\title{
Atuação do psicólogo no Núcleo de Apoio a Saúde da Família: uma experiência em um programa de residência multiprofissional
}

\author{
The practice of the psychologist in the Health \\ Family Support Nucleus: an experience in a \\ multiprofessional residency program
}

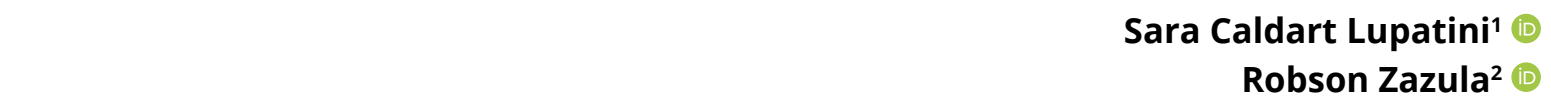

${ }^{1}$ Residência Multiprofissional em Saúde da Família, Universidade Federal da Integração Latino-Americana (Foz do Iguaçu). Paraná, Brasil. saraclpsi@outlook.com

${ }^{2}$ Autor para Correspondência. Instituto Latino Americano de Ciências da Vida e da Natureza, Universidade Federal da Integração Latino-Americana (Foz do Iguaçu). Paraná, Brasil. robson.zazula@unila.edu.br

\begin{abstract}
RESUMO | INTRODUçÃO: A residência é uma modalidade de pós-graduação que se caracteriza pelo treinamento em serviço, tendo como base a aprendizagem pela prática cotidiana. Programas de residência multiprofissional objetivam capacitar profissionais para o exercício qualificado na Atenção Básica de Saúde, a porta de entrada principal do Sistema Único de Saúde (SUS), por meio de metodologias ativas, trabalho em equipe e cuidado humanizado. OBJETIVO: Relatar a experiência e a inserção do psicólogo, enquanto residente, em um programa de residência multiprofissional em Saúde da Família. MÉTODO: Foi realizado o relato de experiência de uma profissional da psicologia enquanto residente do Programa de Residência em Saúde da Família. A presente experiência ocorreu entre março de 2018 e março de 2020 e as seguintes atividades foram descritas: (a) matriciamento, (b) acoIhimentos e atendimento individuais, (c) realização de grupos, (d) educação em saúde, (e) consultas compartilhadas e (f) visitas domiciliares. RESULTADOS: Pode-se afirmar que o processo de formação de profissionais em saúde pública constitui um desafio a gestores, educadores e trabalhadores envolvidos neste processo. Dificuldades em relação ao estímulo simultâneo de habilidades profissionais, interpessoais e humanísticas, bem como senso crítico sobre responsabilidade social são comuns. Ainda assim, a proposta de programas residência é uma oportunidade significativa de aprendizado e contato com outras áreas de saúde. CONCLUSÃO: Diversas limitações puderam ser identificadas ao longo das atividades cotidianas da residência, tal como falta de conhecimento dos profissionais sobre a estratégia de saúde da família e o papel da residência multiprofissional no serviço de saúde.
\end{abstract}

PALAVRAS-CHAVE: Internato não Médico. Psicologia. Atenção Primária em Saúde. Equipe Multiprofissional. Capacitação em Serviço.
ABSTRACT I INTRODUCTION: The residency is a post-graduate modality characterized by in-service training based on learning by daily practice. Multiprofessional residency programs aim to train professionals for qualified exercise in Primary Health Care, the main gateway to the Unified Health System (SUS), through active methodologies, teamwork, and humanized care. OBJECTIVE: To report the experience and the insertion of the psychologist, as a resident, in a multi-professional residency program in Family Health. METHOD: The experience report of a psychology professional was carried out as a resident of the Family Health Residency Program. The present experience took place between March 2018 and March 2020, and the following activities were described: (a) matrix support, (b) Psychological care attendance, (c) groups, (d) health education, (e) consultations, and (f) home visits. RESULTS: It can be said that the process of training public health professionals is a challenge for managers, educators, and workers involved in this process. Difficulties regarding the simultaneous stimulation of professional, interpersonal, and humanistic skills, as well as a critical sense of social responsibility, are common. Even so, the proposal for residency programs is a significant opportunity for learning and contact with other health areas. CONCLUSION: Several limitations could be identified during the daily activities of the residence, such as the lack of knowledge of professionals about the family health strategy and the role of the multi-professional residence in the health service.

KEYWORDS: Non-medical internship. Psychology. Primary Health Care. Multiprofessional team. In-Service Training. 
O Programa Saúde da Família (PSF) surgiu em dezembro de 1993 com uma proposta de reestruturação do sistema público de saúde, organização da atenção primária e substituição dos modelos tradicionais de saúde até então praticados no Brasil. De acordo com Rosa e Labate (2005), o PSF se caracteriza principalmente por ser uma proposta de reorientação do modelo assistencial como uma tentativa de transformar as práticas de atenção à saúde vigentes e o trabalho dos profissionais com foco na Atenção Primária à Saúde (APS), em conformidade com os princípios estabelecidos do Sistema Único de Saúde (SUS). A partir de 2006, o Programa Saúde da Família passa a ser a estratégia permanente de cuidado na APS e, devido à necessidade de continuidade da proposta, passou a ser denominada Estratégia Saúde da Família (ESF) (Dalpiaz \& Stedile, 2011).

No Brasil, a APS é conhecida como porta de entrada dos usuários no SUS. Esta se caracteriza pela descentralização, devendo ser o contato preferencial dos usuários, principal porta de entrada e centro de comunicação destes com toda a rede de atenção à saúde. De acordo com Cezar, Rodrigues e Arpini (2015), a APS se caracteriza pela realização de ações de cunho individual e coletivo que englobam intervenções como foco na promoção, proteção, prevenção, reabilitação, diagnóstico, tratamento e manutenção da saúde. Sua origem remonta ao Modelo Sanitarista de Gestão, com objetivo de substituir o Modelo Hegemônico, hospitalocêntrico e curativo, outrora presente. Além disso, o novo modelo de rede é corroborado pelas diretrizes da Declaração de Alma-Ata (1978), por se tratar de uma metodologia de atenção à saúde essencial e prioritária, fundamentada em tecnologias apropriadas e custo-efetivas (CNRMS, 2012, 16 de abril).

A APS prevê a universalidade, acessibilidade, vínculo, continuidade do cuidado, integralidade da atenção, responsabilização, humanização, equidade e participação social, caracterizando-se um eixo estruturante do Sistema de Saúde (Ministério da Saúde, 2007). Deste modo, situa-se como um caminho para superação das limitações de uma visão fragmentada de sujeito e a construção de um novo modelo não reducionista para realizar as práticas em saúde. A partir de tal concepção, é importante levar em consideração o que de fato é necessário para que este modelo seja implementado e desenvolvido de forma efetiva. A Política Nacional da Atenção Básica (PNAB) elegeu a Saúde da Família como a estratégia prioritária para a organização das ações em saúde, cujos princípios remontam à implantação do Programa de Agentes Comunitário de Saúde, em 1991. Conforme a Política Nacional de Atenção Básica (PNAB; Ministério da Saúde, 2017), a ESF objetiva a reorganização da atenção básica no país, com o propósito de expandir, qualificar e consolidar a APS, visando favorecer uma reorientação do processo de trabalho com maior potencial de aprofundar os princípios, diretrizes e fundamentos da atenção básica. Além disso, objetiva ampliar a resolutividade e impacto na situação de saúde das pessoas e coletividades, além de propiciar uma importante relação custo-efetividade.

Os fundamentos e diretrizes da APS apontam que é necessário (a) possuir território adstrito, de forma a permitir o planejamento, descentralização e desenvolvimento de ações setoriais e intersetoriais; (b) possibilitar o acesso universal e contínuo a serviços de saúde, acolhendo os usuários e promovendo a vinculação e corresponsabilização pela atenção às suas necessidades de saúde; (c) identificar os usuários e com estes desenvolver relações de vínculo e responsabilização entre as equipes e a população, garantindo a continuidade das ações de saúde e a longitudinalidade do cuidado; (d) coordenar a integralidade em seus vários aspectos; e (e) estimular a participação dos usuários como forma de ampliar sua autonomia e capacidade na construção do cuidado à sua saúde e das pessoas e coletividades do território, no enfrentamento dos determinantes e condicionantes de saúde, na organização e orientação dos serviços de saúde a partir de lógicas mais centradas no usuário e no exercício do controle social (Dalpiaz \& Stedile, 2011; Ministério da Saúde, 2017).

Dentro da APS existe o Núcleo de Apoio a Saúde da Família e Atenção Básica (Nasf-AB), um dispositivo estratégico para a melhoria da sua qualidade, que amplia as ações por meio do compartilhamento de saberes e ampliação da capacidade de resolutividade das equipes. Conforme a PNAB (Ministério da Saúde, 2017), o Nasf-AB se caracteriza por ser uma equipe multiprofissional, composta por profissionais com diferentes formações e/ou especialidades, complementar àquelas equipes que atuam na atenção básica, que devem atuar de maneira integrada e apoiando os profissionais das equipes de Saúde da Família. Além disso, é importante que estas equipes compartilhem e apoiem suas práticas em saúde no território sob suas responsabilidades, buscando auxiliá-las no manejo ou resolução de problemas 
clínicos e sanitários, bem como agregando práticas que ampliem suas ofertas, garantindo a longitudinalidade do cuidado e a prestação de serviços diretos à população. Esta equipe é orientada pelo referencial teórico-metodológico do apoio matricial (Chiaverini, 2011; Iglesias \& Avellar, 2019), que busca contribuir para ampliar o alcance das ações, bem como auxiliar na articulação com outros pontos da rede de atenção à saúde, garantindo a continuidade do cuidado aos usuários. A ESF e o Nasf-AB utilizam diversas técnicas e instrumentos para suas atuações, como Projeto Terapêutico Singular (PTS), Programa Saúde na Escola (PSE), visitas domiciliares, trabalhos grupais e matriciamento (Dalpiaz \& Stedile, 2011; Ministério da Saúde, 2017).

\section{A residência multiprofissional como uma nova proposta de paradigma na formação de profissionais de saúde}

A residência em saúde é uma modalidade de ensino de pós-graduação lato-senso com treinamento em serviço, que possibilita ao residente o aprimoramento profissional e a obtenção do título de especialista na área de escolha. Estes programas são destinados às categorias profissionais que fazem parte da saúde, com exceção da medicina, a saber: Biomedicina, Ciências Biológicas, Educação Física, Enfermagem, Farmácia, Fisioterapia, Fonoaudiologia, Medicina Veterinária, Nutrição, Odontologia, Psicologia, Serviço Social e Terapia Ocupacional (Brasil, 2012). Os programas de residência multiprofissional buscam contribuir para a qualificação dos profissionais de modo integral e interdisciplinar em relação àquelas necessidades de saúde da comunidade em que estão, bem como romper com os paradigmas tradicionais em relação à formação de profissionais para SUS (CNRMS, 2012, 16 de abril).

Os programas de Residência Multiprofissional em Saúde (RMS) regulamentados existentes no Brasil se apresentam como uma nova estratégia para a implementação de inovações em políticas públicas. Possuem como objetivo de propor a atuação em saúde de modo centralizado na humanização da assistência, assim como realizar uma implementação efetiva do SUS, conforme seus princípios e diretrizes. Além disso, visa romper com os paradigmas em relação à formação de profissionais para o SUS e contribuir na qualificação da atenção, propondo a integralidade das ações e a realização de trabalho interdisciplinar. Os programas apresentam diferenças em seus desenhos metodológicos, mas em comum defendem a utilização de metodologias ativas e participativas, com educação permanente dos residentes e profissionais do serviço durante todo o processo de formação (Rosa \& Lopes, 2009; Silva, 2018).

No que se refere à formação para o SUS, a RMS produz uma ação inovadora, na medida em que supera a lógica da residência médica, que é historicamente vinculada à especialização médica ou residências uniprofissionais, carecendo de uma discussão coletiva e multiprofissional (Silva, 2018). Uma formação interdisciplinar é uma formação que compartilha saberes, mas mantém integra a particularidade de cada área profissional, possibilitando a socialização de conhecimentos e linguagens no ato da formação e do próprio trabalho em saúde (Monteiro et al., 2019; Silva, 2018).

Em 2005, a RMS é instituída legalmente como modalidade de formação para o SUS, onde se institui também a Comissão Nacional de Residência Multiprofissional em Saúde (CNRMS). Naquele ano existiam cerca de 22 programas de RMS e em 2016, segundo informação da secretária da CNRMS, estavam registrados para avaliação cerca de 1.500 programas de residência uni e multiprofissional. Outro dado, conforme o Relatório do Ministério da Educação (MEC), a partir de 2010 o MEC passa a ter participação no pagamento das bolsas auxílio. Em 2010, foram 414 bolsas, ao passo que em 2014 já havia 3.226 (Monteiro, 2019).

Guido et al. (2012) descreve a residência como um processo de formação em saúde que possui ações inovadoras, em comparação com os modelos tradicionais de ensino e treinamento em serviço presentes nos cursos de graduação e pós graduação. Entretanto, ressalta que alguns aspectos podem ser avaliados como estressores nesse processo, principalmente por não se incluírem no modelo ensino vigente. Dentre estes estressores, destacam-se a realização de ações e atividades em equipe, atividades que envolvam a participação ativa do residente, por meio de fóruns e grupos de discussão, as relações interpessoais estabelecidas com colegas de outras profissões, a responsabilidade de empregar cuidado integral e humanizado, o excesso de trabalho administrativo, excessiva carga de assistencial e de trabalho durante o processo formativo, problemas relacionados à qualidade do ensino e aos ambientes educacionais. $O$ resultado de sua pesquisa com residentes multiprofissionais mostra que os sentimentos de baixa eficiência e produtividade no trabalho prevalecem entre estes, ou seja, o questionamento que 
o profissional faz sobre a sua escolha de profissão, colocando em dúvida a sua aptidão para exercê-la, sentindo-se inadequado pessoal e profissionalmente. Resultados semelhantes foram encontrados por Cahu et al. (2018), que identificaram que profissionais da residência multiprofissional estão em situação elevada de estresse e baixa qualidade de vida.

\section{A psicologia enquanto campo de atuação na Saúde Pública}

Inicialmente, a psicologia não fazia parte da equipe mínima da ESF. Embora o profissional da psicologia fizesse parte da APS desde meados dos anos 1980, mudanças efetivas foram observadas apenas a partir de meados dos anos 2000. De acordo com Cordeiro et al. (2017), a inserção dos psicólogos no SUS iniciouse a partir do ano de 2004, por meio de portarias interministeriais que incentivavam o apoio matricial à ESF. Ainda assim, as mudanças foram pouco significativas, sendo que foi apenas em 2008 que esta categoria foi incluída como uma profissão integrante da APS (Jimenez, 2011; Lima, 2005). Tal inclusão ocorreu devido ao aumento expressivo da demanda por atendimentos com foco na saúde mental na APS (Campos \& Domitti, 2007). De acordo com Dimenstein e Macedo (2012) e Cezar et al. (2015), as práticas da psicologia demonstram uma série de fragilidades no trabalho do psicólogo na APS. Dentre as fragilidades observadas, destacam-se a permanência de um modelo focado na psicoterapia clínica individual, dificuldades para a realização de ações contextualizadas e no trabalho em equipe. Nesse cenário, o papel do profissional da psicologia se faz tão importante. No entanto, a inserção destes profissionais na ESF, ou ainda a inserção da psicologia em programas de residência multiprofissional é algo pouco discutido e abordado na literatura. Considerando a prevalência das doenças e agravos mentais na população geral, os princípios da APS, o papel da APS como porta de entrada do SUS e a frequência de atendimentos relacionados à saúde mental na APS e na ESF, bem como a importância da inserção de profissionais da psicologia e o seu papel na ESF, o objetivo do presente artigo é relatar a experiência e a inserção do psicólogo, enquanto residente, em um programa de residência multiprofissional em Saúde da Família.

\section{Metodologia}

O relato em questão trata-se das experiências de uma psicóloga residente do programa de Residência Multiprofissional em Saúde da Família, gerido pela Universidade Federal da Integração Latino Americana (UNILA) em parceria com a Secretaria Municipal de Saúde do município de Foz do Iguaçu. Por se tratar de um relato de experiência, se caracteriza por descrever uma vivência profissional considerada exitosa (ou não) dentro de um determinado contexto, de modo a contribuir para a atuação de outros profissionais ou para a melhoria do cuidado na saúde pela equipe ou pelo programa de residência. Além disso, é importante destacar que, dada a natureza do presente trabalho, não é possível a reprodução do estudo, uma vez que ele descreve fatos e reflete o cenário em um dado momento.

A presente experiência ocorreu entre março de 2018 e março de 2020. Dentre as atividades descritas no presente relato de literatura, destacam-se: (a) matriciamento, (b) acolhimentos e atendimento individuais, (c) realização de grupos, (d) educação em saúde, (e) consultas compartilhadas e (f) visitas domiciliares. Por se tratar de um relato de experiência, o presente trabalho não foi submetido ao comitê de ética em pesquisa com seres humanos. No entanto, todos os procedimentos éticos previstos, tais como o sigilo e a confidencialidade, foram seguidos na elaboração do presente trabalho. Por fim, é importante destacar que, dado o ineditismo do tema, o presente relato de experiência poderá contribuir na atuação de futuros residentes com formação em psicologia em programas de residência ou até mesmo na formulação de estratégias de atuação na ESF.

\section{Resultados e discussão}

\section{Uma visão geral sobre o programa de residência multiprofissional em Saúde da Família da UNILA e a atuação da psicologia neste contexto}

O programa de residência em Saúde da Família da UNILA é relativamente novo. Iniciou suas atividades em março de 2017, com profissionais graduados em diferentes áreas da saúde, a saber: enfermagem, 
fisioterapia, nutrição, odontologia, bacharel em saúde coletiva e psicologia. De modo geral, o objetivo do programa é formar profissionais de saúde com conhecimentos, habilidades e atitudes para atuar na ESF e Nasf-AB na perspectiva do cuidado integral à saúde em diferentes ciclos de vida familiar, considerando o planejamento estratégico local, a organização do processo de trabalho em equipe e as abordagens interdisciplinares e interpessoais no campo da saúde. Além disso, há um interesse em especial em relação à situação de fronteira e suas respectivas peculiaridades, uma vez que o município de Foz do Iguaçu se localiza em uma região de fronteira trinacional, com o Paraguai e a Argentina (UNILA, 2016).

O Projeto Político Pedagógico (PPC) da Residência Multiprofissional em Saúde da Família da UNILA propõe que o programa possua como perfil de egresso profissionais que possuam uma compreensão ampliada do seu papel no cenário da APS, com capacidade ativa, corresponsável e crítico-reflexiva. Tal perfil prevê ainda que estes profissionais possuam comprometimento com a viabilização de uma prática pautada nos princípios do SUS em sua totalidade, voltado às necessidades sociais, epidemiológicas e de saúde para a população de fronteira. O programa ainda propõe formação crítica e transformadora para o exercício na Saúde da Família, com fundamentos adequados à cada uma das realidades e necessidades. Além disso, propõe que os residentes sejam motivados pela busca permanente de conhecimento, capazes de trabalhar em equipe de forma espontânea e racional, com qualidade e resolubilidade, salvaguardando sempre as especificidades de cada profissão (UNILA, 2016).

Há ainda no PPC uma seção que descreve o perfil do egresso conforme a formação de cada uma das categorias profissionais existentes no programa. No caso específico da Psicologia, é exigido que o profissional esteja apto a desenvolver todas as ações pertinentes ao Nasf-AB, fornecer apoio matricial às equipes e atuar frente às demandas da comunidade, dentro de práticas integrais perpassando por suas diretrizes. Descrições semelhantes são apontadas por Queiroz (2019), que pesquisou a inserção de profissionais psicólogos egressos de programas de residência multiprofissional no Brasil. Entretanto, conforme apontou Cezar et al. (2015), esta inserção ainda é muito tímida e, em muitos casos, a atuação da psicologia está restrita à permanência de um modelo clínico individual, com pouca contextualização nos determinantes de saúde e dificuldades na realização de trabalhos em equipes multidisciplinar.

Uma das atividades descritas como parte do perfil do egresso dos profissionais da psicologia é o matriciamento. Segundo o Ministério da Saúde (MS; Chiaverini, 2011; Ministério da Saúde, 2014), o matriciamento consiste em um arranjo organizacional que visa outorgar suporte técnico-pedagógico em determinadas áreas do conhecimento para as equipes responsáveis pelo desenvolvimento de ações na APS com a população do território. De acordo com o Caderno 39 da AB, o trabalho do Nasf-AB é orientado pelo apoio matricial. Este mesmo documento traz diversos recursos para que esse apoio seja implementado, com destaque para a realização de reuniões de matriciamento com determinada periodicidade, estudos de caso, atendimento domiciliares, grupos e atividades coletivas e atendimentos individuais específicos (Ministério da Saúde, 2014). Do ponto de vista da psicologia, o matriciamento pode ser compreendido como um excelente recurso para a inserção do profissional desta área de atuação, em especial na condução de ações com foco na saúde mental. Neste cenário, o matriciamento pode ser compreendido como recurso de construção de ações na saúde mental também junto às comunidades, no território onde as pessoas vivem e circulam, por meio da proposta de encontros produtivos, sistemáticos e interativos entre as equipes de APS e as equipes de saúde mental do serviço (Campos, 1999; Chiaverini, 2011; Iglesias \& Avellar, 2019).

Embora esta seja uma prática prevista nas atribuições e responsabilidades também do psicólogo dentro do Nasf-AB e ser trazida como a prevalente nas diretrizes e recomendações, é uma das ações que foram pouco desenvolvidas e incorporadas às práticas do psicólogo neste contexto. De modo geral, poucas atividades de matriciamento foram realizadas na APS de Foz do Iguaçu como um todo, durante o período de realização da residência e abrangendo a área da psicologia, e mais especificamente no contexto da saúde mental, uma vez que reuniões periódicas de matriciamento, independentemente da área, não eram práticas corriqueiras no serviço de saúde do município. Por outro lado, espaços como reuniões de equipe da UBS ou reuniões sobre a Saúde do Trabalhador eram utilizados para abordar questões associadas à saúde mental, tais como fluxos de trabalho ou informações acerca 
das ações que poderiam ser desenvolvidas, caso fossem necessários. Constantemente havia discussões e divergências entre os diferentes profissionais da equipe sobre o que a prática do matriciamento consistia, bem como em suas aplicações, demonstrando a necessidade de maior exploração deste campo de prática, não apenas pela psicologia, mas pelas demais profissões da saúde.

Outra prática corriqueira dos psicólogos no Nasf-AB eram os acolhimentos psicológicos, uma modalidade breve de atendimento demandada em situações conflitivas emergentes e urgentes, por meio de atendimento para alívio de sintomas de modo momentâneo (Furigo et al., 2008; Sonneborn \& Weba, 2013), bem como atendimentos clínicos individuais. Essas eram as atividades que ocupavam a maior parte da agenda dos profissionais e residentes da psicologia, o que ocorria devido a existência de listas de espera de usuários do território que procuravam a UBS para esta modalidade de atendimento. Em alguns momentos também eram acolhidos pacientes encaminhados pelas equipes de referência. A prática clínica dentro do espaço de saúde público se caracterizava por ser a atividade profissional mais realizada pelos psicólogos, conforme experiência cotidiana e relato de profissionais da área em reuniões de equipe no município. Relatos semeIhantes também são identificados na literatura, conforme apontado por Cezar et al. (2015).

O método de acolhimento, implementado na equipe do Nasf-AB em que a primeira autora do presente relato de experiência atuou, consistia em agendar atendimentos individuais, a partir das listas de espera disponíveis da unidade básica de saúde (UBS), por meio de encaminhamentos de outros profissionais do NasfAB ou demanda espontânea. De acordo com as diretrizes gerais desses acolhimentos, cada usuário poderia ser atendido por até cinco sessões, que possuíam como objetivo avaliar as queixas e possibilidades de intervenção breves e, caso necessário, eventuais encaminhamentos. Eram considerados atendimentos prioritários nesta lista de espera: gestantes, idosos, queixas relacionadas a lutos e perdas, e risco de suicídio. Na prática, muitos dos casos permaneciam por maior tempo, passando a ser incorporados na rotina de atendimentos psicológicos individuais regulares. Em alguns momentos excepcionais, o acolhimento era realizado de modo emergencial, ou seja, algum outro profissional acionava uma das psicólogas que estivessem presentes na UBS, de modo a realizar uma escuta inicial sobre a demanda apresentada na consulta de outra especialidade (em especial durante consultas com os profissionais médicos). Nestes casos, umas das profissionais ou residentes da psicologia realizavam o acolhimento de modo imediato, independentemente da atividade previamente agendada para aquele momento.

Ainda em relação aos atendimentos clínicos individuais ou acolhimentos, dentre as principais demandas desta modalidade destacam-se queixas psiquiátricas como transtornos ansiosos e depressivos, bem como a alta frequência de queixas relacionadas à de ideação e tentativas de suicídio. O principal ponto negativo dessa prática era a sua pouca abrangência desta, uma vez que as listas de espera eram bastante extensas, por meio de demandas que deveriam ser atendidas em sua maioria de modo individual, fatores que inviabilizavam a realização do acolhimento da forma como ele é trazido pela literatura. Devido à grande quantidade de usuários que buscavam tais atendimentos, especialmente por meio de demandas espontâneas, no momento do contato telefônico muitos usuários diziam já ter solucionado aquilo que os afligiam no momento da solicitação do atendimento, ao passo que em outros casos era nítido que os sintomas ou condições haviam se agravado. Além disso, a realização dessas ações, dada a elevada demanda, dificultavam a busca por ações com foco na promoção da saúde, o que deveria ser o principal objetivo da APS.

Outra prática prevista e realizada durante o período da residência foram os grupos operativos com os usuários da APS do território. De modo geral, grupos operativos se caracterizam pela possibilidade de uma nova elaboração de conhecimento e integração, nos quais a aprendizagem se caracteriza por ser um processo contínuo em comunicação e interação com o outro (Bastos, 2010). Estes foram pontuais e liderados por outros profissionais, com a participação dos residentes da psicologia.

Dentre os grupos operativos desenvolvidos, o grupo "Vida Mais Leve", idealizado e gerido pela nutricionista, no qual os participantes possuíam demandas relacionadas à alimentação e perda de peso. O objetivo principal desta ação era promover hábitos alimentares saudáveis e a busca pelo emagrecimento. O grupo foi planejado para ser realizado em oito encontros semanais, sendo que em cada um destes um tema específico era trabalhado. E em um desses encontros a Psicologia trabalhava questões relacionadas à 
ansiedade, depressão, emoções e sentimentos bem como a inter-relação destes com o comportamento alimentar. Nestas inserções, houve pouca adesão dos participantes nos encontros por diversos motivos, mesmo com demonstrações de interesse dos participantes com o tema e o conteúdo trabalhado. Em resumo, dada essas condições, acredito que houve pouco espaço para se alcançar a produção de um conhecimento. Outras propostas de intervenção, como um grupo mais específico e focado realizado pela psicologia, chegaram a ser pensados, porém não foram desenvolvidos durante o decorrer da residência devido a dificuldades operacionais e ao excesso de demanda de outras atividades.

Uma segunda proposta implementada foi o planejamento e a implementação de grupos de tabagismo. Estes foram grupos estruturados com números de sessões, temáticas e estratégias baseadas no manual e protocolo propostos pelo Programa Nacional de Tabagismo, desenvolvido pelo MS. Para a realização destes grupos, houve a participação de diferentes profissionais que atuavam no Nasf-AB, cuja participação ocorria de modo alternado, conforme as temáticas e atividades que eram desenvolvidas. Além desse grupo, um terceiro grupo, de caráter aberto e focado na Saúde do Trabalhador também foi executado. Estes grupos ocorriam semanalmente com os colaboradores da UBS em que a residência foi realizada e, de modo geral, suas sessões eram divididas em dois momentos: um primeiro momento com foco em atividades laborais, e um segundo momento no formato de uma roda de conversa, coordenados pela preceptora e residente em psicologia, por meio da abordagem de temas e assuntos pedidos pelo grupo.

As atividades de educação em saúde ocorriam com grande frequência no território de atuação da residente. Estas ações se caracterizam por intervenções junto aos usuários da comunidade com o objetivo de produzir reflexão promover conhecimento sobre prevenção de doenças e promoção da saúde. Uma das intervenções realizadas foi a implementação do PSE, que se caracteriza por ser uma política intersetorial entre o MS e o MEC instituída em dezembro de 2007, com objetivo de contribuir para o fortalecimento da promoção e prevenção de doenças e agravos, de modo a proporcionar um desenvolvimento saudável em crianças e adolescentes por meio da integração entre saúde e educação (Farias et al., 2016).
No caso específico de Foz do Iguaçu, a gestão municipal possui um colaborador encarregado exclusivamente pela coordenação do programa, incluindo o envolvimento de profissionais de diferentes áreas de atuação. Durante o primeiro ano da residência, houve maior envolvimento das residentes da psicologia nesta ação em todas as suas etapas, desde a escolha de tema, passando pelo levantamento da demanda, planejamentos e preparações, execução, e avaliação dos principais resultados. Conforme a proposta e prerrogativas do PPC do programa de residência e da ESF, esta ação foi desenvolvida de modo interdisciplinar, envolvendo não apenas residentes da psicologia, mas também residentes de outras áreas como enfermagem e fisioterapia (UNILA, 2016). A ação em questão abordou temas relacionados à sexualidade entre alunos do quarto e quinto ano de uma das escolas de ensino fundamental do distrito de saúde no qual a residência foi desenvolvida, bem como em outros distritos, conforme demanda específicas destes. As ações ocorreram por meio da realização de dois a quatro encontros com cada uma das turmas destas escolas, composta por aproximadamente 30 alunos.

Os resultados planejados para o PSE podem ser considerados de médio a longo prazo, especialmente por se tratar de temas que envolvam mudanças de comportamento e atitudes em relação a um determinado tema ou assunto. No entanto, alguns resultados foram observados imediatamente às intervenções realizadas, especialmente por meio do relato dos educadores e responsáveis pelas escolas no que se refere às falas e posturas dos alunos quando os temas trabalhados surgiam em outras atividades no ambiente escolar, reproduzindo tópicos durante a intervenção realizada imediatamente antes da realização destas atividades. Além disso, no próprio intervalo entre os encontros era nítido o maior entendimento e atenção que os alunos apresentavam em relação aos temas trabalhados. No que se refere às limitações, a falta de tempo para estender intervenções a impossibilidade de iniciá-las ainda mais precocemente foi um dos pontos identificados ao final das intervenções. Outra limitação está relacionada à compreensão e adesão dos pais ao trabalho da temática, mesmo tendo sido realizada uma reunião com os mesmos para explicar sobre o trabalho poucos compareceram. 
Outras ações de educação em saúde desempenhadas na residência foram realizadas diretamente nas UBSs com os usuários de grupos com pacientes com doenças crônicos, tal como hipertensão, nos grupos chamados de 'Hiperdia', com frequência semanal, e os grupos de insulino-dependentes, com frequência mensal. Os grupos de caminhada e "Turma da Coluna" ocorriam duas vezes na semana e possuía um público-alvo heterogêneo, composto, em sua maioria, com pessoas com idade média acima dos 50 anos e portadores de doenças crônicas. Profissionais e os residentes da psicologia participaram das intervenções nos grupos de caminhada semanalmente, caminhando junto aos usuários, com o principal objetivo de criar e formar vínculos junto a esta comunidade, bem como a realização do processo de territorialização. Estes eram espaços ainda foram utilizados para desenvolver ações para temáticas de educação em saúde, tais como: Maio Amarelo, Agosto Azul, Setembro Amarelo, Outubro Rosa, luta antimanicomial, combate ao abuso de crianças e adolescentes, informações sobre o que o SUS e como alguns fluxos funcionam, temas relacionados à saúde mental, bem-estar, qualidade de vida, alimentação, exercícios de relaxamento ou alívio de dores específicas, controle da pressão arterial, dados antropométricos, ou ainda outros que temas solicitados ou de interesse dos usuários.

Ainda seguindo as temáticas mensais ou dias de específicos com foco em determinadas temáticas, também ocorriam intervenções na sala de espera ou em outros espaços da comunidade, por meio de eventos marcados com as equipes de referência dentro da UBS, como “Dias D" para determinados temas. Essa ação foi intitulada Cine Saúde e consistia na exibição de um filme era para comunidade e os profissionais dentro da sala de espera da UBS e, em sequência uma discussão à cerca das percepções encontradas no filme e a relação com a saúde. Em outras ações, desenvolvidas em um dos sábados em que a UBS foi aberta ao público, foi disponibilizado diversos atendimentos ao mesmo tempo para homens, sem necessidade de agendamentos prévios, sendo o acolhimento psicológico um deles. Dentre as ações adicionais destacam-se a realização de uma gincana no estacionamento de uma das UBS em que a residência era realizada, abordando temas como a promoção da qualidade de vida.

A realização de consultas compartilhadas foi uma das atividades que trouxeram maior proximidade de entendimento e vínculos entre os profissionais e equipes, bem como entre estes com a população.
As consultas compartilhadas se caracterizam por ser um instrumento de trabalho que privilegia a comunicação transversal entre os membros da equipe de saúde, possibilitando a abordagem de um problema de modo transversal, sob diferentes olhares e perspectivas (Luz et al., 2016). No caso das atividades da residência, estas ocorriam principalmente com a enfermeira e médica, majoritariamente em atendimentos de puericulturas e pré-natal.

A experiência enquanto residente durante as consultas compartilhadas podem ser avaliadas como enriquecedora, uma vez que foi por meio destas que foi possível conhecer melhor os profissionais e a população para a qual a ação da psicologia foi dirigida, com bastante troca e vinculação. Durante consultas já agendadas pelas equipes de referência era apresentado e explicado aos usuários o papel de cada um dos profissionais e, caso julgassem pertinente, poderiam questionar algo caso desejassem durante a consulta (e.g., como estava se sentindo com a gravidez ou naquele momento, como o companheiro ou familiares participavam das suas experiências, com o que estava tendo dificuldades naquele momento/processo). No que se refere à psicologia, cabia à residente, dentre outras coisas, avaliar e observar questões a respeito da vinculação materna, sinais de baby blues, depressão pós-parto ou qualquer outro sintoma emocional, quando outro profissional da equipe estava em dúvidas sobre a situação emocional de alguma paciente. Caso alguma paciente se mostrasse mais fragilizada, uma sessão de acolhimento psicológico poderia ser realizada pelos profissionais da psicologia.

Embora as visitas domiciliares apareçam como uma das atividades constantes na ESF, assim se estendendo ao Nasf-AB, esta não era uma ação na qual a psicologia estava plenamente inserida. Relatos apontam a importância da atuação do profissional da psicologia nesta prática no Nasf-AB e, de acordo com Oliveira et al. (2017), a maioria das visitas domiciliares possuem alguma relação com saúde mental e devem ser realizadas com usuários com impossibilidade de locomoção, que não podem sair de casa. No caso específico nos territórios no qual a residência estava sendo conduzida, em poucas oportunidades estas atividades foram realizadas pelos profissionais da psicologia e tinham como principal objetivo atender demandas específicas e emergenciais dos usuários, identificados a partir de relato prévio dos demais profissionais da equipe. Durante a residência, houve a vivência desta atividade apenas durante a realização do diagnóstico 
no acompanhamento da visita de outros profissionais. Assim como os autores citados anteriormente, esta prática necessita ser repensada quanto aos objetivos e modos de realização pelos profissionais da psicologia, uma vez que possuem elevada importância, dada a frequência de problemas com foco na saúde mental em pessoas com impossibilidade de locomoção bem como seus respectivos cuidadores (Oliveira et al., 2017).

\section{Considerações finais}

A residência é uma modalidade de ensino relativamente nova, sua implementação e concretização ainda trilha um caminho de ajustes, apesar desta proporcionar um cenário ideal para a preparação do profissional para a APS. O processo de formação de profissionais em saúde pública constitui um desafio a gestores, educadores e trabalhadores envolvidos neste processo, sempre havendo complexidade quanto ao estímulo simultâneo de habilidades profissionais, interpessoais e humanísticas, bem como senso crítico sobre responsabilidade social. Ainda assim, a residência é uma oportunidade significativa de aprendizado e contato com outras áreas de saúde. Um deles trata-se do trabalho multiprofissional e interdisciplinar tanto em relação às equipes de referência quanto a possibilidade de trabalhos com os demais residentes. No entanto, ao analisarmos a residência do ponto de vista da psicologia, em particular o papel do psicólogo e a inserção deste nas ESF, pode-se afirmar que o desafio é um pouco maior. Uma das maiores dificuldades vivenciadas foi a inclusão da psicologia dentro do trabalho das equipes de ESF. Essa dificuldade pode estar intimamente relacionada com dificuldade de os profissionais da psicologia trabalharem de modo contextualizado e em equipe, fruto da formação unidisciplinar e tradicional, com foco no trabalho individual (Cezar et al. 2015).

Apesar dos avanços obtidos no modelo de atenção à saúde, é possível observar a presença do antigo modelo biologista, reproduzimos muitas vezes na graduação e ainda não superado, dificultando a inserção de discussões, reflexões e modificações para além do saber médico. O mesmo pode ser dito em relacionado à atuação do psicólogo na APS. Observase claramente a presença do modelo clínico individual, tão característico da psicologia. Mesmo havendo uma clara tentativa para atualizar esse modelo, envolvendo outras estratégias de atuação, observase ainda resquícios, tal como nos acolhimentos psicológicos ou mesmo nos atendimentos clínicos individuais, realizados com os usuários que buscam esta modalidade de atendimento. Para a superação dentro da profissão e do próprio programa da residência é importante ressaltar para que os profissionais estejam sensíveis às percepções e vivências, colocando em ação práticas que estejam na contramão do modelo hegemônico, desde a teoria até a prática. Essa superação deve ser não apenas dos profissionais da equipe, mas também dos profissionais da psicologia.

Um ponto de destaque, e enfatizado por Cezar et al. (2015), é a carência de formação específica na psicologia com foco em saúde coletiva. A grande maioria dos cursos de graduação em psicologia não possuem disciplinas ou componentes curriculares com foco em saúde pública, o que se reflete tanto na atuação do psicólogo na APS, quanto na concepção dos demais profissionais acerca deste profissional dentro das equipes. Embora estes sejam imprescindíveis dentro das equipes, o foco permanece em um modelo clínico e individual, reproduzindo um modelo hospitalocêntrico. Devido à carência na formação dos profissionais da psicologia, tanto aqueles que atuam no serviço, quanto dos residentes, há a manutenção dessa perspectiva, pela carência de modelos de atuação.

A residência procura produzir reflexões quantos as efetividades e melhorias do SUS como um todo, e que assim cumpre uma das suas possibilidades emergentes, que é a da problematização da realidade. A dificuldade de contextualização da psicologia a diversos cenários de prática, somado à carência na formação acadêmica, dificultam este processo do ponto de vista da psicologia. As demais profissões que atuam na APS, de modo geral, e na ESF, de modo mais específico, possuem dificuldades semelhantes. Entretanto, devido a uma maior fundamentação teórica e técnica no campo da saúde coletiva, conseguem lidar de modo mais fácil com esta dificuldade, fato que não pode ser observado em relação à psicologia, conforme identificado durante a experiência do presente trabalho. Nesta concepção, principalmente no início da inserção dos residentes, estes foram vistos como um grupo à parte e não integrantes da equipe, até mesmo no sentido de fiscalização e cobrança de algumas ações, sendo este um fato a ser superado para alcançar o trabalho em equipe, se dando pela própria construção de vínculos. 
Talvez um dos grandes pontos limitadores que contribuem para essas realidades é a própria falta de estrutura dos serviços, sendo físicas e de capital humano. Além disso, a eminente falta de conhecimento sobre no que consiste um programa de residência e como este se dá pela maioria dos colaboradores do serviço de saúde do município. Especificamente dentro da área de saúde de mental, várias foram as dificuldades enfrentadas pela residente, tais como a falta de profissionais específicos da área nos serviços público de saúde, de modo geral, e na APS, de modo mais específico. Além disso, dentre os demais profissionais de saúde que atuam na APS foi nítida a falta de preparo e empoderamento para lidar com pacientes com demandas relacionadas a saúde mental, muitas vezes resultando em encaminhamentos ou condutas equivocadas que podem gerar agravos em longo prazo.

\section{Agradecimentos}

Os autores agradecem ao apoio da Secretaria Municipal de Saúde de Foz do Iguaçu, Paraná, Brasil e da Secretaria do Programa de Residência Multiprofissional em Saúde da Família. Agradecimento especial aos Ministério da Saúde/ Ministério da Educação pela concessão da bolsa durante o período da residência (março 2018 a fevereiro 2020).

\section{Contribuições dos autores}

Lupatini SC participou da concepção, descrição dos resultados, delineamento interpretação dos resultados e redação e Zazula $R$ participou da concepção, descrição dos resultados, interpretação dos resultados, redação e encaminhamento do artigo científico.

\section{Conflitos de interesses}

Nenhum conflito financeiro, legal ou político envolvendo terceiros (governo, empresas e fundações privadas, etc.) foi declarado para nenhum aspecto do trabalho submetido (incluindo, mas não se limitando a subvenções e financiamentos, participação em conselho consultivo, desenho de estudo, preparação de manuscrito, análise estatística, etc.).

\section{Referências}

Alves, R. B, Bruning, N. O., \& Kohler, K. C. (2019). "O Equilibrista”: Atuação do Psicólogo no NASF no Vale do Itajaí. Psicologia: Ciência e Profissão, 39, e186600. https://doi. org/10.1590/1982-3703003186600

Bastos, A.B.B.I. (2010). A técnica de grupos-operativos à luz de Pichon-Rivière e Henri Wallon. Psicólogo informação, 14(14), 160-169. http://pepsic.bvsalud.org/scielo.php?script=sci arttext\&pid=S1415-88092010000100010\&lng=pt\&tIng=pt

Cahú, R.A.G., Santos, A.C.O., Pereira, R.C., Vieira, C.J.L., \& Gomes, S.A. (2014). Estresse e qualidade de vida em residência multiprofissional em saúde. Revista Brasileira de Terapia Cognitiva, 10(2), 76-83. https://dx.doi.org/10.5935/1808$\underline{5687.20140013}$

Campos, G.W.S. (1999). Equipes de referência e Apoio Especializado Matricial: um ensaio sobre a reorganização do trabalho em saúde. Ciência e Saúde Coletiva, 4(2), 393403. https://doi.org/10.1590/S1413-81231999000200013

Campos, G. W. S., \& Domitti, A. C. (2007). Apoio matricial e equipe de referência: Uma metodologia para gestão do trabalho interdisciplinar em saúde. Cadernos de Saúde Pública, 23(2) 399-407. https://doi.org/10.1590/50102$\underline{311 \times 2007000200016}$

Cezar, P. K., Rodrigues, P. M., \& Arpini, D. M. (2015). A Psicologia na Estratégia de Saúde da Família: Vivências da Residência Multiprofissional. Psicologia: Ciência e Profissão, 35(1), 211 224. https://doi.org/10.1590/1982-3703000012014

Chiaverini, D.H. (Org.), Gonçalves, D. A., Ballester, D., Tófoli, L. F., Chazan, L. F., Almeida, N., \& Fortes, S. (2011). Guia prático de matriciamento em saúde mental. Ministério da Saúde, Centro de Estudo e Pesquisa em Saúde Coletiva. http://bvsms.saude.gov.br/bvs/publicacoes/guia_pratico matriciamento saudemental.pdf

Cordeiro, S. N., Reis, M. E. B. T., Spagiari, N. T. B., \& Adamowski, W. D. (2017). Contribuições da Psicologia à Residência Multiprofissional em Saúde da Mulher: Relato de Experiência. Revista Polis e Psique, 7(3), 100-115. https:// doi.org/10.22456/2238-152X.72447

Dalpiaz, A.K., \& Stedile, N.L.R. (2011, 23-26 de agosto). Estratégia saúde da família: reflexão sobre algumas de suas premissas [Apresentação em conferência]. V Jornada internacional de políticas públicas, São Luiz, MA, Brasil. http://www. joinpp.ufma.br/jornadas/joinpp2011/CdVjornada/ JORNADA_EIXO_2011/IMPASSES_E_DESAFIOS_DAS POLITICAS_DA_SEGURIDADE_SOCIAL/ESTRATEGIA_SAUDE_ DA FAMILIA REFLEXAO SOBRE ALGUMAS DE SUAS PREMISSAS.pdf 
Farias, I. C., Sá, R.M.F., Figueiredo, N., \& Menezes-Filho, A. (2016). Análise da intersetorialidade no Programa Saúde na Escola. Revista Brasileira de Educação Médica, 40(2), 261-267. https://dx.doi.org/10.1590/1981$52712015 v 40 n 2 e 02642014$

Furigo, R.G.P.L, Sampedro, K.M., Zanelato, L.S., Foloni, R.F., Ballalai, R.C., \& Ormrod, T. (2008). Plantão psicológico: uma prática que se consolida. Boletim de Psicologia, 58(129), 185-192. http://pepsic.bvsalud.org/pdf/bolpsi/ v58n129/v58n129a06.pdf

Guido L.A., Silva, R.M., Goulart, C.T., Bolzan, M.E.O., \& Lopes, L.F.D. (2012). Síndrome de Burnout em residentes multiprofissionais de uma universidade pública. Revista da Escola de Enfermagem da USP, 46(6), 1477-1483. http:// dx.doi.org/10.1590/S0080-62342012000600027

Iglesias, A., \& Avellar, L.Z. (2019). Matriciamento em Saúde Mental: práticas e concepções trazidas por equipes de referência, matriciadores e gestores. Ciência e Saúde Coletiva, 24(4), 1247-1254. http://dx.doi.org/10.1590/141381232018244.05362017

Jimenez, L. (2011). Psicologia na Atenção Básica à saúde: demanda território e integralidade. Psicologia e Sociedade, 23(spe), 129-139. https://dx.doi.org/10.1590/s0102$\underline{71822011000400016}$

Lima, M. (2005). Atuação psicológica coletiva: uma trajetória profissional em unidade básica de saúde. Psicologia em Estudo, 10(3), 431-440. https://dx.doi.org/10.1590/S141373722005000300011

Luz, A.R., Vianna, M.S., Fátima-Silqueira, S.M., Silva, P.C., Chagas, H.A., Figueiredo, J.O., Mortimer, F.M., \& Starke, A.C. (2016). Consulta compartilhada: uma perspectiva da clínica ampliada na visão da residência multiprofissional. Revista Eletrônica de Gestão e Saúde, 7(1), 270-281. https:// periodicos.unb.br/index.php/rgs/article/view/3419

Portaria $n^{\circ} 2.436$, de 21 de setembro de 2017. Aprova a Política Nacional de Atenção Básica, estabelecendo a revisão de diretrizes para a organização da Atenção Básica, no âmbito do Sistema Único de Saúde (SUS). https:// bvsms.saude.gov.br/bvs/saudelegis/gm/2017/ prt2436_22_09_2017.html

Ministério da Saúde. (2014). Núcleo de apoio à saúde da família - Volume 1: Ferramentas para a gestão e para o trabalho cotidiano. Cadernos da Atenção Básica, 39. http://bvsms. saude.gov.br/bvs/publicacoes/nucleo apoio saude familia_cab39.pdf
Monteiro, M.S.F., Ferreira, I.P., Galvão, S.S.C., Borges, S.C.R., Silva, F.R., Oliveira L.R.L., Cardoso, R.F., Costa, J.B., Marques, S.E.S., Mendes, B.C.S., Figueira, V.K.S., \& Santos, J.P.V. (2019). Residência Multiprofissional em Saúde da Família e suas contribuições para os serviços de saúde: revisão integrativa. Revista Eletrônica Acervo Saúde, 24, e519. https://doi.org/10.25248/reas.e519.2019

Oliveira, I.F., Amorim, K.M., Paiva, R.D., Oliveira, K.S., Nascimento, M.N., \& Araújo, R.L. (2017). A atuação do psicólogo nos NASF: desafios e perspectivas na atenção básica. Temas em Psicologia, 25(1), 291-304. http://dx.doi.org/10.9788/ TP2017.1-17Pt

Queiroz, A.H.A.B. (2019). Política de inserção de psicólogos egressos das residências multiprofissionais em saúde na docência [Tese de Doutorado, Universidade Federal do Rio Grande do Norte]. Repositório Institucional UFRN. https:// repositorio.ufrn.br/jspui/handle/123456789/28341

Rosa, S.D., \& Lopes, R.E. (2009). Residência multiprofissional em saúde e pós-graduação lato sensu no Brasil: apontamentos históricos. Trabalho, Educação e Saúde, 7(3), 479-498. https://doi.org/10.1590/S1981$\underline{77462009000300006}$

Rosa, W. A. G, \& Labate, R. C. (2005). Programa saúde da família: a construção de um novo modelo de assistência. Revista Latino-Americana de Enfermagem, 13(6), 1027-1034. https:// dx.doi.org/10.1590/S0104-11692005000600016

Resolução CNRMS n. 2, de 13 de abril de 2012. Dispõe sobre Diretrizes Gerais para os Programas de Residência Multiprofissional e em Profissional de Saúde. http://portal.mec.gov.br/index.php?option=com docman\&view=download\&alias=15448-resol-cnrms-n213abril-2012\&ltemid $=30192$

Silva, L.B. (2018). Residência Multiprofissional em Saúde no Brasil: alguns aspectos da trajetória histórica. Revista Katálysis, 21(1), 200-209. https://doi.org/10.1590/198202592018v21n1p200

Sonneborn, D., \& Werba, G.C. (2013). Acolher, cuidar e respeitar: contribuição para uma teoria e técnica do acolhimento em saúde mental. Conversas Interdisciplinares, 8(3), 4-16. http://www.periodicos.ulbra.br/index.php/ci/article/ view/3953/pdf

Universidade Federal da Integração Latino Americana. (2016). Projeto Político Pedagógico: Programa de Residência Multiprofissional em Saúde da Família. https://unila.edu.br/ sites/default/files/files/PPC\%20-\%20Resid\%C3\%AAncia\%20 Multiprofissional.pdf 